\title{
Clinical Reasoning to Advance Medication Safety by Senior Nursing Students
}

\author{
Elizabeth Domm \\ University of Regina, liz.domm@uregina.ca \\ Bonnie Raisbeck \\ Saskatchewan Polytechnic, bonnie.raisbeck@saskpolytech.ca \\ Megan Pearce \\ University of Regina and Saskatchewan Polytechnic, pearce857@gmail.com
}

Follow this and additional works at: https://qane-afi.casn.ca/journal

Part of the Other Nursing Commons

\section{Recommended Citation}

Domm, Elizabeth; Raisbeck, Bonnie; and Pearce, Megan (2021) "Clinical Reasoning to Advance Medication Safety by Senior Nursing Students," Quality Advancement in Nursing Education - Avancées en formation infirmière: Vol. 7: Iss. 1, Article 5.

DOI: https://doi.org/10.17483/2368-6669.1255

This Article is brought to you for free and open access by Quality Advancement in Nursing Education - Avancées en formation infirmière. It has been accepted for inclusion in Quality Advancement in Nursing Education - Avancées en formation infirmière by an authorized editor of Quality Advancement in Nursing Education - Avancées en formation infirmière. 


\section{Clinical Reasoning to Advance Medication Safety by Senior Nursing Students}

\section{Cover Page Footnote}

The authors wish to acknowledge the participation of the senior nursing students of the SCBScN program and funding from the University of Regina and Saskatchewan Polytechnic, Collaborative Grant. Les auteures aimeraient souligner la participation des étudiantes en sciences infirmières de $4 \mathrm{e}$ année du programme SCBScN ainsi que le financement reçu grâce à une bourse de collaboration de la University of Regina et de la Saskatchewan Polytechnic. 
As soon-to-be registered nurses, senior nursing students are expected to use clinical reasoning to safely and competently administer medications to patients in clinical practice (Saskatchewan Registered Nurses Association, 2015; 2019). Clinical reasoning was described as the "use [of] clinical judgment and critical thinking to make decisions based on both knowledge and experience" (Herron et al., 2016, p. 329). Medication safety was most recently defined as "freedom from preventable harm with medication use" (Institute of Safe Medication Practices Canada, 2007, para 23). Nursing students acquire knowledge in nursing education programs about how to critically think and use clinical reasoning to safely administer medications in the clinical settings (Bourbonnais \& Caswell, 2014; Canadian Association of Schools of Nursing, 2011; Stec, 2016). Levett-Jones et al. (2010) developed a clinical reasoning educational model for nursing students to use to identify at-risk patients. Recently, Preston et al. (2019) reported that while nursing student survey respondents valued pharmacological education, respondents did not perceive their knowledge of pharmacological theory was readily used to support safe medication administration in clinical practice. Moreover, there appears to be a gap in knowledge regarding how senior nursing students use clinical reasoning to support medication safety with patients in clinical settings. The purpose of this qualitative study was to develop an understanding as to how senior nursing students used clinical reasoning to manage and support medication safety in their final clinical practice experiences and whether students used the Levett-Jones et al. clinical reasoning model.

\section{Background}

To clearly understand senior nursing students' documented use of clinical reasoning to support medication safety, we searched databases CINAHL, MEDLINE, and PubMed, and Google Scholar for the central concepts of senior nursing students, clinical reasoning, and medication safety. Nurse scholar and nurse educator Alfaro-LeFevre (2017) described that nurses use clinical reasoning as "dynamic and interrelated [cognitive processes] regulated by standards, policies, procedures, and individual [jurisdictional] practice acts" (p. 144) to make clinical judgments and decisions when responding to patient health needs. Levett-Jones et al. (2010) developed an educational model for nursing students to identify cues and implement correct nursing actions with acutely ill patients at risk of deterioration using "the five rights of clinical reasoning" (p. 517). Jessee and Tanner (2016) developed a clinical coaching inventory to measure senior-level prelicensure nursing students' development of clinical reasoning as outcomes of clinical coaching by faculty and preceptors. Bae et al. (2019) sought to further develop undergraduate nursing students' clinical reasoning following students' participation in patient simulation experiences that were not specific to medication administration by implementing a simulation debriefing protocol. Lee and Quinn (2019) reviewed the nursing literature and found that nursing education programs frequently taught nursing students about medication administration using simulation experiences and online learning modules with technological support.

Preston et al. (2019) explored nursing students' perceptions of the importance of pharmacological education for safe medication administration and reported that while students valued the knowledge gained about the actions of medications and the nursing process, students did not find didactic pharmacological theoretical education was readily transferred to clinical practice. Cleary-Holdforth and Leufer (2020) surveyed senior nursing students to determine their perceptions of their competence for medication administration and found that most respondents reported feeling ill-prepared to administer medications safely owing to a lack of adequate pharmacological knowledge and opportunities to administer medications in clinical settings. 
Moloney et al. (2020) sought senior nursing students' perspectives about their educational preparation for medication management and reported that students perceived their nursing education to have a pivotal role in their understanding of safe medication management. No research reports indicated that nursing students were asked to describe how they applied clinical reasoning to achieve medication safety for patients in their clinical practice. The problem addressed in this study is how do senior nursing students use clinical reasoning to support medication safety when administering medications to patients in clinical settings?

\section{Method}

A qualitative descriptive case study design (Yin, 2014) was used to gain an understanding of how senior nursing students described their critical thinking, clinical reasoning, judgment, and actions when managing and administering medications in clinical settings. A qualitative case study design was appropriate to acquire an understanding of senior nursing students' descriptions of their experiences, the richness of which might not be captured by using a different approach. The LevettJones et al. (2010) educational model developed to guide nursing students' clinical reasoning to detect deterioration in the condition of at-risk patients was used as the conceptual model for this study. This study was approved by university and institutional research ethics boards and nursing education program administration. The study was completed in 2019.

\section{Sample}

The study sample comprised 13 senior nursing students in their final practicums. The researchers recruited participants using posters, emails, and snowball sampling in one western Canadian nursing program. Potential participants contacted researchers to indicate interest in study participation and schedule an interview. Participants read and signed consents before participating in approximately one-hour interviews.

\section{Data Collection}

Data collection took place during confidential individual interviews with participants. The researchers asked senior nursing student participants the following questions:

1. "Can you tell me about a time during your senior practicum where you assessed a patient or client, and based on your nursing knowledge, you were concerned about medication safety?"

2. "Can you describe what your critical thinking and clinical reasoning were in that situation that led you to act to support medication safety while implementing safe patient-centred care in your nursing practice?"

All interviews were audio recorded and transcribed verbatim. Researchers read and cleaned transcripts soon after each interview to exclude potentially identifying information. Data collection concluded after the 13th interview owing to the fact that the researchers noted that new perspectives of clinical reasoning to support medication safety were not apparent.

\section{Data Analysis}

Inductive reasoning was used to analyze the data for meanings and to construct themes (Woo, 2019). Research team members individually read the transcripts with an initial focus on extracting examples of students' descriptions of how they used clinical reasoning to support medication safety. Transcripts were coded line by line using NVIVO12 software. Quotations were sorted and displayed in table form, revealing potential codes, clusters, and categories. The research 
team met regularly to discuss the meanings embedded in participants' stories, and the codes, categories, and patterns emerging from data. Researchers identified themes representing key findings that illustrated senior nursing students' clinical reasoning to support medication safety.

\section{Trustworthiness}

Study approval by university and institutional research ethics boards was granted before the study commenced. Confidentiality of participant information was maintained and informed written consent was obtained before participation in the study. Quotations from anonymized participants were provided to demonstrate the credibility of data, the stability of findings over time from students in diverse clinical settings, and the rigour of methods that suggest results could be comparable if this study were to be replicated with senior nursing students in similar contexts. Research team members independently read transcripts, had access to interview records to verify the accuracy of transcribed data, and arrived at similar conclusions about the meanings and themes in data, thereby establishing confirmability. Findings may be transferable to other nursing programs in which senior nursing students' clinical reasoning to support medication safety is assessed in clinical practice placements with preceptors.

\section{Findings}

All volunteer participants were female senior nursing students engaged in their final clinical practice rotation of the nursing program with a preceptor. Six themes were interpreted from the data that described students' clinical reasoning to support medication safety: (1) administering medications safely by recognizing and clustering cues, (2) administering medications safely to the right patient in the context of care, (3) administering medications safely by determining the correct action, (4) administering medications safely to patients for the right reason, (5) reflecting on clinical reasoning experiences to support medication safety, and (6) recognizing unit culture impacts on reasoning about medication safety.

\section{Theme 1: Administering Medications Safely by Recognizing and Clustering Cues}

Participants described how they used clinical reasoning by initially recognizing and then clustering cues regarding the patient and medication administration context, including patient medication histories, changes in vital signs, level of consciousness, and response to medications. Recognizing and clustering cues were important considerations for students as they reasoned whether it was safe to administer patients' prescribed medications. While participants provided many examples of noticing cues by assessing the patient's history and physical presentation, students identified that there were times when they missed subtle cues. After reflecting on the patient's situation, students described being able to identify the patient's condition associated with recognizing and clustering cues the next time. A student who completed a final clinical practicum on a palliative care unit described how she engaged in clinical reasoning by recognizing and responding to clustered cues to ensure the patient's pain was relieved and medications were safely administered:

I am on a palliative unit, and on palliative a lot of times the patients are kind of unresponsive. And so we have to focus a lot on micro expressions in those cases. So looking for like furrowed brows, really tense, or like kind of rigid posture ... A lot of times if I walk into a patient's room and they are in the state where they are unresponsive, if I notice like furrowed brows, that would indicate to me that they are having pain and so I would look to see if they have any PRN [as needed] pain medications and when the last scheduled 
medications were given ... and administer those and kind of assess after 30 minutes or so, see if it has worked, and if not do it again. (Participant 12)

This student described using clinical reasoning when recognizing and clustering patient cues and using cues to safely and adequately manage pain for patients on a palliative care unit. Similarly, another participant noted how important it was to recognize patient cues, know the intended effects and possible adverse effects for the patient with each medication being administered:

Knowing that that's my responsibility as a nursing student and nurse to kind of know the expected outcomes of [medications], but also the adverse effects, I guess. So, knowing that it's supposed to lower her blood pressure but at that time lowering her blood pressure wasn't safe. (Participant 6)

By understanding the effects of medication and recognizing patient cues, this participant described how she used clinical reasoning to support medication safety by not administering the medication at that time.

\section{Theme 2: Administering Medications Safely to the Right Patient in the Context of Care}

Senior nursing student participants' abilities to identify patients at particular risk of an adverse medication event supported their safe medication practice. A student participant described how she used clinical reasoning by double-checking each patient's identity to safely identify that the right patient was receiving the right medication before administering medications:

Whenever I was giving medications, I always made sure ... I [was] thinking of ... the right patient and I ... [identified each patient] by their last names and check their name bands. Like I could visually see who they were, but I ... don't want to get in the habit of assuming it is the right patient by like doing just a visual check. (Participant 9)

The student described reasoning to form a habit of double-checking patient identity to safely administer medications to the right patient in the context of care. In contrast, another participant verbally illustrated how in a previous clinical experience she did not identify the right patient before administering a medication, and this resulted in a medication error:

I did have an error once ... it makes you really, really cautious ... I had three patients and two of them had high blood pressure, both on Ramipril of the same dose and the doctor ordered to give another $5 \mathrm{mg}$ of Ramipril and my instructor just handed me the [medication administration record] MAR and the medication and said go give this med. I assumed it was for the patient who had the higher blood pressure ... and it was actually ordered for the one who had the lower blood pressure. I noticed once I was putting the MAR back into the book at the medication cart because I was like the [patient] MAR's already in here and my heart just sank. Because they were both on the same dose, but I just didn't even look. [Note: student and instructor monitored patient and followed hospital protocol for a medication error.] (Participant 7)

In this situation, the participant described how her experience reinforced the importance of clinical reasoning to administer medications safely by checking the patient's identity with the physician's order and medication administration record before administering the medication.

A participant described a situation in which she identified a patient who was at risk for complications from diabetes, and how she reasoned to manage the patient's care appropriately: 
[The patient had] Type 1 diabetes ... he presented like a diabetic ketoacidosis ... during the night we had gone in to check his blood sugar ... and it was 1.7. And so we started the hypoglycemic protocol and it hadn't come up ... so then we decided to phone the doctor and he said bolus D5W 50 [5\% dextrose in water, 50 milliliters intravenously] ... and then it [blood glucose level] came up. (Participant 10)

The senior nursing student recognized that this patient was at risk for complications from diabetes. She took the initiative to check his blood glucose and acted to address the patient's hypoglycemia promptly based on her clinical reasoning.

\section{Theme 3: Administering Medications Safely by Determining the Correct Action}

Participants described using clinical reasoning in many situations to interpret what the correct action and intervention were in patient situations with the medications that were ordered. A participant gave an example of the importance of administering medications safely by determining the correct action before and after administration of the medication that potentially affects a patient's vital signs:

If you give a medication that affects the heart rate and you only do ... vitals every four [hours], you need to make sure that in half an hour or whatever, however long it takes for that medication to kick in, you go back and check and make sure that it is actually doing its job, you need to make sure that you note those kinds of things. (Participant 2)

This participant described administering medications safely includes recognizing that the timing of assessments is crucial. Similarly, a participant working with a preceptor recalled a time when she used clinical reasoning to determine the correct nursing actions for an elderly patient with a slow heart rate who had a scheduled medication prescribed to slow the patient's heart rate:

I checked the [patient's] pulse. It was low. Checked it manually. Still low and [this patient was] was ordered $25 \mathrm{mg}$ of metoprolol, so I knew that that would further reduce her pulse and I was not comfortable giving it. I went back and checked the MAR ... There was nothing in the nursing notes that said anything ... so I went to my preceptor and told him my findings and he asked what I would do. I said I would hold it and put it on the doctor's ask list for tomorrow, what he wants to do with it or give us some parameters or something. So, I didn't give it to her that night, and I felt good about that. (Participant 13)

This participant described how she used her knowledge of the actions of this medication to assess and reassess the patient, then discussed her concerns with her preceptor before administering the medication. In this instance, the student used clinical reasoning to determine that it was unsafe to administer the medication to this patient at this time based on her knowledge of the pharmacotherapeutics of the medication. The student described how withholding the medication and notifying the patient's physician supported medication safety for that patient in the context of care.

Participants described how clinical reasoning and critical thinking guided their actions as nursing students especially when administering medications. One participant described the importance of applying critical thinking as a future registered nurse:

Critical thinking is an essential component in being a successful nurse. It allows you to use your knowledge, experience, and gut to achieve the best outcome for your clients. With critical thinking and medication administration, they are so important and really do go together. (Participant 4) 
This participant described how knowledge, critical thinking, and clinical experience contributed to the development of her clinical reasoning about medication safety in practice.

\section{Theme 4: Administering Medications Safely to Patients for the Right Reason}

Participants described using clinical reasoning to determine whether appropriate medications were administered to patients for the right reasons. A participant recalled providing care for a patient who had been experiencing a fever for several days. The etiology of the patient's fever was not overtly being investigated, and the patient was being treated with Tylenol. This participant described her reasoning that Tylenol was not being administered for the right reason as Tylenol could mask symptoms of the patient's illness:

[The patient's] temperature was 39.4 , that's way too high for a healthy man ... something was ringing off in my mind and then the LPN [licensed practical nurse] that was standing beside me said, "Oh, we have just given him Tylenol for that [his fever]." And I went okay, how many times have we had to give him Tylenol? "Oh, we've given quite a bit now. It just doesn't seem to be bringing his temperature down." And in my mind I'm [thinking] Tylenol is not, that's not the right drug. [Patient was later diagnosed with sepsis.] (Participant 1)

The participant described that she reasoned that Tylenol was not the single right medication to repeatedly administer to this patient, because it was not the right medication to address what was causing the patient's fever. Another participant described that while a medication prescribed for a patient in her care was being given for the right reason she used clinical reasoning to question if the scheduled time for the medication to be administered was right given the patient's condition:

This patient was ... ordered [prescribed] on top of the $40 \mathrm{mg}$ of furosemide to add on another $60 \mathrm{mg}$ of furosemide ... I was questioning the amount of furosemide that we were giving and also the timing. Once the medication activates [the patient] will start peeing and that's fine, but at night ... it creates a lot of risks for the patient ... they were guided mobility ... so they weren't the kind of patient that I wanted them to get out of bed right after they woke up and try and find their way to the bathroom. (Participant 9)

The senior nursing student used clinical reasoning to determine that administering this medication at the time it had been ordered could endanger the patient's safety. She then acted to address this concern with the physician, who changed the scheduled time for this medication.

\section{Theme 5: Reflecting on Clinical Reasoning Experiences to Support Medication Safety}

Barbagallo (2019) described "reflective practice [as] the ability to learn from experiences and to understand and develop practice from said reflection" (p. 160). Participants described reflecting on their experiences and the context of care when they administered medications. One participant reflected on her role as a nursing student administering medications in a long-term care facility:

I just found that medication safety, it can really depend on the context too because in the long-term care setting ... like the reasoning behind our care was mostly what's therapeutic for the residents and how can this be resident-focused? ... Like how can we actually make this a better way for the resident so that it is safe, and it is therapeutic? ... I know sometimes the best thing for people isn't always medications too. It's not always like pushing the pills on them. (Participant 11) 
This student reflected on her clinical reasoning to support medication safety by evaluating her reasoning about the safety and therapeutic nature of administering medications for residents. Reflection was regarded by this participant as an integral component of clinical reasoning for safe and therapeutic medication administration. Another participant described reflecting on a clinical situation when a patient who was receiving an anti-seizure medication was not showing improvement in seizure activity. The participant described how she questioned the method in which the medication was being administered:

This one patient ... we are giving this $5 \mathrm{~mL}$ [millilitres] of anti-seizure medication ... in the deltoid. Yeah, and then I thought 'that's not right' ... and so we looked it up ... it is like 1 to 2 [millilitres], oh, that's probably why the medication isn't working because we have been giving too much medication in a small muscle. (Participant 11)

Although the participant stated she did not initially recognize that administering this volume of medication was too great for a small muscle, she later reflected that this could be the reason why administering the medication was not reducing the patient's seizure activity. Through this reflection, she reasoned that "too much medication" was administered in the deltoid.

\section{Theme 6: Recognizing Unit Culture Impacts on Reasoning about Medication Safety}

Participants recognized the clinical unit culture surrounding medication administration practices of nurses differed at times from the best medication administration practices learned by students in nursing school. Some participants described observing nurses' medication preparation and administration practices that supported medication safety. Participants described recognizing the importance of learning how medication dosages were calculated and administered in unit contexts such as the neonatal intensive care unit before proceeding to administer medications as they had learned in their nursing education program. A participant working on an adult patient unit reported that nurses on that unit did not routinely double-check insulin dosages each time before administration as students are required to do:

I really found push back with the insulin. With having the double-check ... I had a substitute preceptor. She's like, "Why don't you just give it? We just sign the double-check at the end of the shift." And I'm like, "because I am a student and I need to come show you this." I didn't say it but I thought, Because insulin can kill you. So, like I really ticked her off every time and ... well, they were all talking about it because they just couldn't believe that every time I had to give insulin I needed to double-check the dose with a nurse, and I would come ... find somebody. (Participant 13).

This student used clinical reasoning to administer medication safely in practice while recognizing that unit medication administration practices were different from what she had learned. Another participant discussed how she used clinical reasoning about medication safety after learning that nurses on one unit did not necessarily check patients' blood pressures immediately before administering medications that lower patients' blood pressure:

I noticed that the blood pressure meds, they (nurses on unit) don't take their blood pressures prior to giving them. And so I said to my preceptor, like you guys don't take his blood pressure before giving ... the blood pressure meds? What if it is low and you bottom them out more? So we did actually go and take it and it was within normal limits. (Participant 10) 
This participant demonstrated how she used clinical reasoning and questioned a practice of nurses on one unit to maintain what she believed was safe medication administration practice. While participants recognized variations in some unit medication administration practices from what students learned in nursing school that could impact their clinical reasoning about medication safety for patients, students did not generally describe that they asked nurses working on units about medication administration practices.

\section{Discussion}

Findings in this study provide an indication as to how senior nursing student participants described they used clinical reasoning to achieve medication safety and reflected about medication safety in clinical practice. Themes included participants' use of critical thinking and clinical reasoning to recognize and cluster patient cues to inform timely actions to administer medications to the right patient for the right reasons while recognizing the impact unit culture had on their reasoning. The Levett-Jones et al. (2010) educational model was developed for nursing students' use when endeavouring to discern and reason about actions for patients at risk of deterioration. As this study focused on how senior nursing students used clinical reasoning to support medication safety in clinical practice, findings indicated that senior nursing students described using similar but not identical "rights of clinical reasoning" (Levett-Jones et al., 2010, p.517) and illuminated that their critical thinking and clinical reasoning to support medication safety was intensely influenced by patient needs and contexts of care. The findings from this study are further compared with other study findings focused on nursing students' clinical reasoning when administering medications.

Preston et al. (2019) conducted a qualitative study in which nursing students were asked how their pharmacological education influenced their ability to administer medications safely. Preston et al. reported that students stated that assessments and pharmacological knowledge supported medication safety, however, classroom learning about pharmacological theory was not easily transferred to the clinical setting. Similarly, Cleary-Holdforth and Leufer (2020) found that senior nursing students reported feeling inadequately equipped to administer oral medications to patients, based on their insufficient pharmacological knowledge and lack of opportunity to administer medications to patients during their program. Moloney et al. (2020) found senior nursing students recognized that nursing education was important for safe medication management, however, students were not asked to describe how they applied clinical reasoning to achieve medication safety in clinical practice. In the current study, senior nursing students articulated how they used their knowledge of medications when assessing and recognizing patient cues, which supported their ability to administer medications safely to the right patient at the right time for the right reasons and advocate for medication safety for patients.

Lee and Quinn (2019) found that medication administration safety in undergraduate nursing education programs was most often taught using technology, simulation, and learning modules. Bae et al. (2019) reported that simulation was imperative to assist nursing students to develop clinical reasoning, discussed the impact of simulation on nursing students' clinical reasoning, and the value of reflection and debriefing following a simulated clinical event. In addition, Bae et al. (2019) reported that nursing students perceived their ability to reason and form connections between class content and their simulation experience improved when opportunities were available for debriefing and reflection. In this study, senior students reflected that the culture of the clinical setting in which they were working with a preceptor included accepted approaches 
to nursing care of patients, preceptors' points of view and influence, assessments of patient cues, nursing actions employed, and how they could have reasoned to promote medication safety.

In a study conducted by Herron et al. (2016), nursing students identified that simulation experiences supported their developing clinical reasoning abilities as students felt safe making mistakes when nursing instructors were present, and no real patients were at risk. However, Herron et al. reported students expressed that clinical reasoning was best learned in clinical and simulation settings. These findings were also supported by a study conducted by Oostra et al. (2019), wherein students expressed that their clinical reasoning, developed through writing a clinical assignment and learning about clinical reasoning, was enhanced in simulation and clinical experiences. In the current study, participants expressed that it was essential for students to use clinical reasoning to administer medications safely in practice settings where patients could be placed at substantial risk if students could not or did not reason like a nurse.

Fisher and Kiernan (2019) conducted a qualitative study and found unit culture had an impact on nursing students' willingness to voice their concerns and that the students' ethical values ultimately determined if students spoke up when they noticed something that was not being carried out according to best practice. In addition, Fisher and Kiernan found that rather than challenge the unit culture, students would often conform in an effort to gain acceptance. In the current study, participants described how their reasoning and actions to maintain medication safety were often guided by their moral values and focus on safe medication administration to benefit patients in their care.

One implication of these results indicates that nursing student participants engaged in, reflected on, and described how they used critical thinking and clinical reasoning to support medication safety for patients and manage their clinical practice while working alongside preceptors. An implication is that educational strategies may need to be modified to enhance student opportunities to engage in clinical reasoning, reflection, and collaboration with nurses in practice about medication safety. Furthermore, nursing education programs and clinical settings for nursing students might develop shared expectations for nursing students' clinical reasoning to ensure medication safety. Further research is needed to provide evidence as to how nursing students learn to apply clinical reasoning to support medication safety in different patient care contexts and unit cultures. In addition, research is required to explore and understand how nurses working within specific clinical practice settings use clinical reasoning to support medication safety and how senior nursing students can learn from nurses in practice.

A limitation of the current study is the small sample of female participants recruited from one nursing education program; however, data saturation was achieved, indicating the sample size was appropriate for this qualitative descriptive case study. In addition, participants' recollections may have been influenced by the study's purpose, which focused on how they used clinical reasoning to support medication safety.

\section{Conclusion}

Senior nursing students used clinical reasoning to safely administer medications by recognizing, clustering, and responding to patient cues, interpreting reasons, and implementing actions to administer medications safely to patients in the context of care. Nursing students also reflected how their actions and unit culture influenced medication safety. A recommendation based on these findings was that nursing education program faculty seek to enhance opportunities for nursing students to describe how they use critical thinking, clinical reasoning, and clinical 
Quality Advancement in Nursing Education - Avancées en formation infirmière, Vol. 7, Iss. 1 [2021], Art. 5

judgement when administering medications in practice settings and perhaps collaborate to understand how unit culture influences students' use of clinical reasoning to safely administer medications. 


\section{References}

Alfaro-LeFevre, R. (2017). Critical thinking, clinical reasoning, and clinical judgement: A practical approach (6th ed.). Elsevier.

Bae, J., Lee, J., Jang, Y., \& Lee, Y. (2019). Development of simulation education debriefing protocol with faculty guide for enhancement clinical reasoning. BMC Medical Education, 19(1), 1-7. https://doi.org/10.1186/s12909-019-1633-8

Barbagallo, M. S. (2019). Completing reflective practice post undergraduate nursing clinical placements: A literature review. Teaching \& Learning in Nursing, 14(3), 160-165. https://doi.org/10.1016/j.teln.2019.02.001

Bourbonnais, F., \& Caswell, W. (2014). Teaching successful medication administration today: More than just knowing your "rights." Nurse Education in Practice, 14(4), 391-395. https://doi.org/10.1016/j.nepr.2014.03.003

Canadian Association of Schools of Nursing. (2011). Baccalaureate education and baccalaureate programs: Position statement. https://casn.ca/wpcontent/uploads/2014/10/BaccalaureatePositionStatementEnglishFinal.pdf

Cleary-Holdforth, J., \& Leufer, T. (2020). Senior nursing students' perceptions of their readiness for oral medication administration prior to their final year internship: A quantitative descriptive pilot study. Dimensions of Critical Care Nursing, 39(1), 23-32. https://doi.org/10.1097/DCC.0000000000000401

Fisher, M., \& Kiernan, M. (2019). Student nurses' lived experience of patient safety and raising concerns. Nurse Education Today, 77, 1-5. https://doi.org/10.1016/j.nedt.2019.02.015

Herron, E., Sudia, T., Kimble, L., \& Davis, A. (2016). Prelicensure baccalaureate nursing students' perceptions of their development of clinical reasoning. Journal of Nursing Education, 55(6), 329-335. https://doi.org/10.3928/01484834-20160516-05

Institute of Safe Medication Practices Canada. (2007). Definition of terms. http://www.ismpcanada.org/definitions.html

Jessee, M., \& Tanner, C. (2016). Pursuing improvement in clinical reasoning: Development of the clinical coaching interactions inventory. Journal of Nursing Education, 55(9), 495504. https://doi.org/10.3928/01484834-20160816-03

Lee, S. E., \& Quinn, B. L. (2019). Incorporating medication administration safety in nursing education: A literature review. Nurse Education Today, 72, 77-83. https://doi.org/10.1016/j.nedt.2018.11.004

Levett-Jones, T., Hoffman, K., Dempsey, J., Jeong, S., Noble, D., Norton, C., \& Hickey, N. (2010). The "five rights" of clinical reasoning: An educational model to enhance nursing students' ability to identify and manage clinically "at risk" patients. Nurse Education Today, 30(6), 515-520. https://doi.org/10.1016/j.nedt.2009.10.020

Moloney, M., Kingston, L., \& Doody, O. (2020). Fourth year nursing students' perceptions of their educational preparation in medication management: An interpretive phenomenological study. Nurse Education Today, 92. https://doi.org/101016/j.nedt.2020.104512 
Oostra, K., Astle, B., \& Meyerhoff, H. (2019). Clinical reasoning on an assignment: Baccalaureate nursing students' perceptions. Quality Advancement in Nursing Education, 5(1). https://doi.org/10.17483/2368-6669.1152

Preston, P., Leone-Sheehan, D., \& Keys, B. (2019). Nursing student perceptions of pharmacology education and safe medication administration: A qualitative research study. Nurse Education Today, 74, 76-81. https://doi.org/10.1016/j.nedt.2018.12.006

Saskatchewan Registered Nurses Association. (2015). Medication management for RNs: A patient centred decision-making framework. https://www.srna.org/wpcontent/uploads/2017/09/Medication_Management_for_RNs_FINAL_2015_09_03_Rev_ 2015_09_30_Web.pdf

Saskatchewan Registered Nurses Association. (2019). Registered nurse entry-level competencies. https://www.srna.org/wp-content/uploads/2019/09/RN-Entry-Level-Competencies2019.pdf

Stec, M. (2016). Health as expanding consciousness: Clinical reasoning in baccalaureate nursing students. Nursing Science Quarterly, 29(1), 54-61. https://doi.org/10.1177/0894318415614901

Woo, K. (2019). Polit \& Beck Canadian essentials of nursing research (4th ed.). Wolters Kluwer.

Yin, R. Y. (2014). Case study research: Design and methods (5th ed.). Sage. 